\title{
MONITORING OF THE FATIGUE OF D16AT ALLOY ACCORDING TO THE CHARACTERISTICS OF DEFORMATION SURFACE PATTERN
}

\author{
S. R. Ignatovich ${ }^{1,2}$ and S.S. Yutskevich ${ }^{1}$
}

\begin{abstract}
It is shown that the deformation pattern is formed under cyclic loads and develops on the surface of the cladding layer of a structural aluminum alloy. The quantitative parameters of the deformation pattern, namely, the saturation, roughness, and plastic strains on the surface, serve as indicators of damage to the material. The experimental dependences of the analyzed parameters on the operating time (number of loading cycles) can be used for the creation of new methods aimed at the prediction of the limit state of structural elements of the aircraft structures.
\end{abstract}

Keywords: deformation pattern, interference profilometry, fatigue, parameters of the deformation pattern.

The processes of fatigue damage in metals connected with the formation and evolution of the dislocation structure, plastic loosening, and microfracture are localized in the surface layer. More intense plastic deformation of the surface as compared with the bulk of the material enables us to interpret the surface layer as an independent functional subsystem determining the specific features of the local plastic deformation and fracture of the material as a whole [1]. Thus, the surface can play the role of a specific indicator of the degree of accumulated damage, and the assessment of its state can be used as a method of diagnostics of the fatigue damage to the entire structure and the exhaustion of its load-bearing capacity.

As one of promising directions in the investigation of plastic deformation of the surface, we can mention the development of a procedure of monitoring of the engineering state of structures in the course of operation. The sensors (reference specimens) in the form of thin plates or films made of readily deformable materials (e.g., of single-crystal aluminum) are pasted to the most loaded regions of the structure and bear all in-service force actions together with the structure [2]. The history of in-service loading of the monitored structural element specifying the degree of its damage manifests itself in the form of typical traces of the local plastic deformation of the surface of a sensor, namely, slip lines, slip strips, extrusions, intrusions, etc. This means that the deformation pattern (DP) forms on the surface.

In aircraft structures, sheet aluminum alloys coated with a thin cladding layer of commercially pure aluminum are used as structural materials. Under loading, the DP is formed on the surface of the cladding layer and can readily be recorded by optical devices. In this case, the procedure of monitoring of the exhaustion of service life of structural elements can be realized by using the criterion of saturation of the DP (equal to the relative area of the analyzed region covered with traces of plastic deformation) [3]. The specific features of this method are described in $[4,5]$. The quantitative estimation of the DP according to its area, i.e., in the two-dimensional case, is not exhaustive. It is necessary to study the quantitative characteristics of DP in the three-dimensional case $[6,7]$.

\footnotetext{
${ }^{1}$ National Aviation University, Kiev, Ukraine.

${ }^{2}$ Corresponding author; e-mail: ignatovich@nau.edu.ua.
}

Translated from Fizyko-Khimichna Mekhanika Materialiv, Vol.47, No.5, pp.60-65, September-October, 2011. Original article submitted March 17, 2011. 
In what follows, we analyze specific features of the formation and evolution of the DP on the surface of the cladding layer under the conditions of cyclic loading of an aluminum alloy.

\section{Material and Method of Investigations}

Flat specimens of D16AT structural aluminum alloy were cyclically loaded in a BiSS Bi00-202V servohydraulic installation by zero-to-compression stress cycles $\left(\sigma_{\min }=0\right)$ with given value of the maximum stress $\sigma_{\text {min }}$. The surface of the specimens in the as-delivered state was covered with a cladding layer of commercially pure aluminum $50 \mu \mathrm{m}$ in thickness. In the central part of the specimen, we drilled a hole with a diameter of $4 \mathrm{~mm}$ modeling a rivet hole in the structure of the aircraft skin. The surface region adjacent to the hole was polished with a diamond paste.

Under cyclic loading, the specimens were periodically removed from the installation to check the DP. This was performed on a surface $(225 \times 170 \mu \mathrm{m}$ in size $)$ neighboring with the hole in the zone of stress concentration. In each specimen, there were four regions of this kind (two regions on each side of the specimen). The regions of the cladding layer on the surfaces of the specimens were investigated with the help of an interference optical nanoprofilometer [8] used to record the optical images of the surface with traces of the DP, realize their digital processing, and determine the 3D-topography of the surface. Moreover, the degree of saturation of the DP, as well as the roughness and plastic deformation of the surface were also recorded.

Saturation of the DP. In the images obtained with the help of an optical microscope (which is a part of the profilometer), the DP is observed in the form of dark regions (Fig. 1a) corresponding to higher convexities of the surface pattern obtained with the help of the profilometer (Fig. 1b). These images were transformed into monochrome (black-and-white) images and used to determine the total area of the DP $(S)$ in the automatic mode. For the quantitative assessment of the degree of saturation of the DP, we use the formula

$$
D=\frac{S}{A}
$$

where $A$ is the area of the investigated region.

Roughness. The development of the DP in the direction normal to the surface was characterized by the changes in the roughness $R_{a}$ equal to the mean deviation of the absolute values of the surface profile $z_{i}$ from the median line (Fig. 2a):

$$
R_{a}=\frac{1}{n} \sum_{i=1}^{n}\left|z_{i}\right|,
$$

where $n$ is the number of points of measurement of the profile.

We split the investigated region into 320 lines $170 \mu \mathrm{m}$ in length and determine the value of $R_{a i}$ along each line by formula (2) (Fig. 2b). The roughness of the region is found by averaging the value of $R_{a i}$ over all lines. The evolution of roughness of the analyzed region under cyclic loading is characterized by the increment of the parameter $R_{a}$ :

$$
\Delta R_{a}=R_{a N}-R_{a 0},
$$



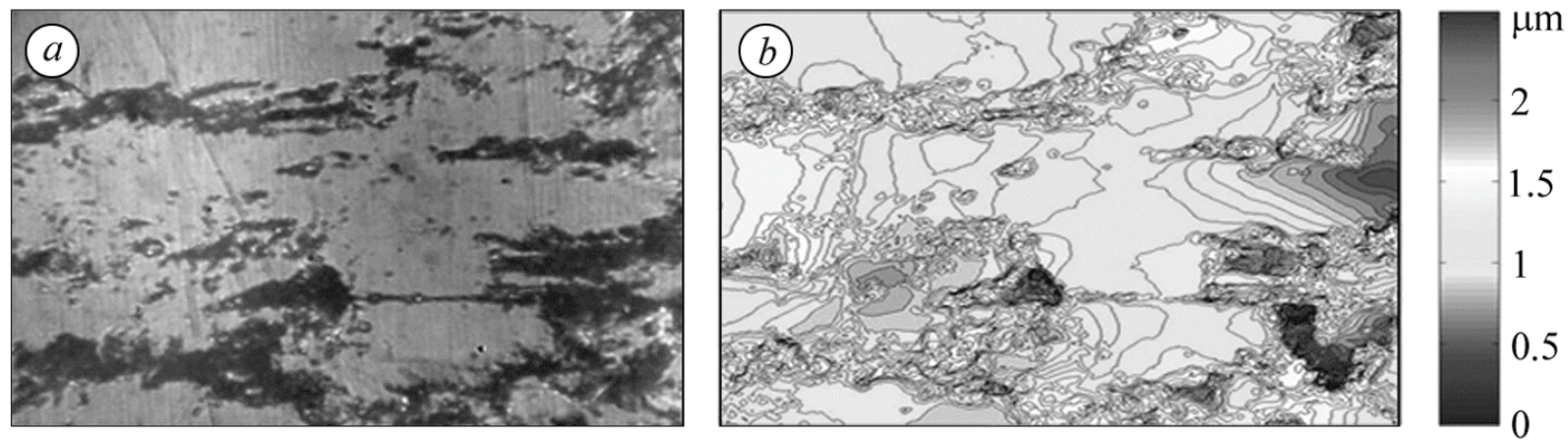

Fig. 1. Digital image $(\times 500)($ a) and 3D topography (b) of a part of the surface with traces of the deformation pattern.
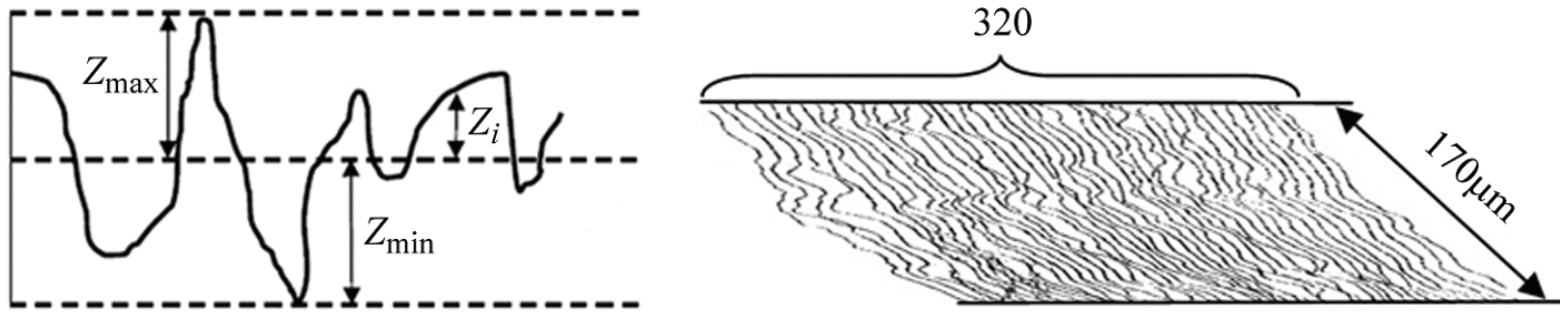

Fig. 2. Computational schemes used for the evaluation of roughness $R_{a}$ along the $i$ th line (a) and in the analyzed part of the surface (b).

where $R_{a 0}$ and $R_{a N}$ are the values of roughness prior to (initial) and after $N$ loading cycles.

Plastic Strains. As the surface pattern changes, the area of the surface increases:

$$
\Delta A=A_{N}-A_{0},
$$

where $A_{0}$ and $A_{N}$ are, respectively, the areas of the surface prior to and after $N$ loading cycles. To find the area of the surface, we represent the projection of the inspected area onto the plane $x, y$ in the form of a grid with $320 \times 240$ nodes. Each of these nodes is associated with the value of height of the surface pattern $z_{i}$ determined by the profilometer (Fig. 3). The surface is replaced by a collection of triangles with vertices whose coordinates correspond to the values of $z_{i}$. The area of the entire surface is computed by finding the sum of the areas of triangles. By analyzing the changes in this area, we determine the level of plastic strains on the surface:

$$
\varepsilon_{a}=\frac{\Delta A}{A_{0}}
$$

\section{Results and Discussion}

Saturation of the DP. The process of local microplastic deformation of the surface of the cladding layer begins from the first loading cycles. We distinguish three stages of the evolution of saturation of the DP under cyclic loading:

In the initial stage of operation, the rate of changes in the parameter $D(1)$ is relatively low. 

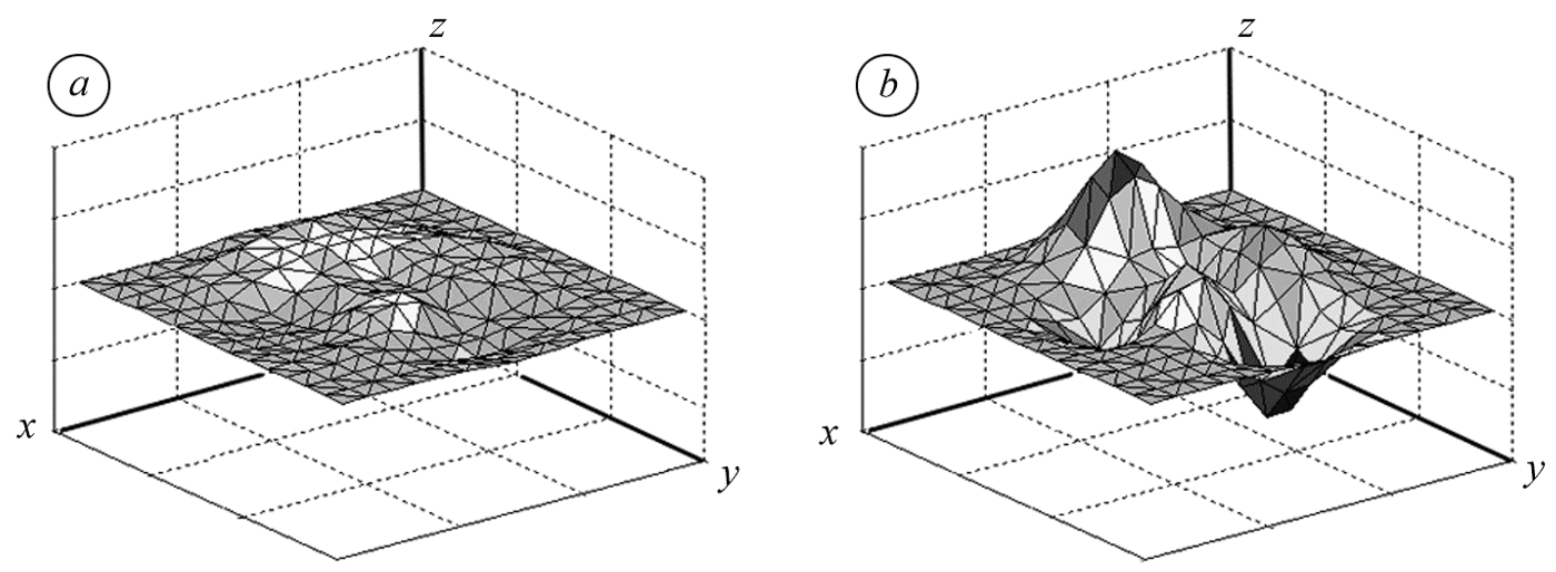

Fig. 3. Evaluation of the surface area in the intact state $A_{0}$ (a) and after $N$ loading cycles $A_{N}$ (b).

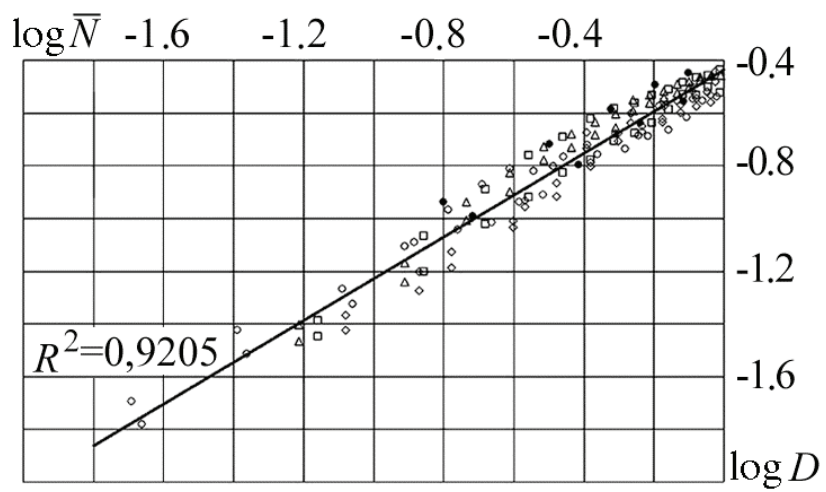

Fig. 4. Dependence of the saturation of the DP on the number of loading cycles prior to the formation of a crack for various values of $\sigma_{\max }:(\bigcirc) 60 \mathrm{MPa},(\square) 80 \mathrm{MPa},(\triangle) 100 \mathrm{MPa},(\diamond) 130 \mathrm{MPa},(\bullet) 150 \mathrm{MPa}(R$ is the correlation coefficient).

Then the intensity of accumulation of the DP increases and, finally, decreases. The last stage of development of the DP corresponds to the stabilization of the degree of saturation of the surface microplastic deformation.

To compare the results obtained for five different values of the maximum stress in a cycle $(60,80,100,130$, and $150 \mathrm{MPa}$ ), the dependences of $D$ on $N$ are reduced to the relative number of loading cycles $\bar{N}=N / N_{f}$, where $N$ is the current number of loading cycles and $N_{i}$ is the number of cycles prior to the initiation of a 0.5 -mm-long crack in the inspected region. It is shown that the variations of the degree of saturation of the DP as a function of the relative number of loading cycles do not depend on the acting stresses (Fig. 4) and can be described by the following power function:

$$
D=0.3656 \bar{N}^{0.8}
$$

Dependence (6) indicates that the saturation of the DP is a generalized characteristic of the number of loading cycles to the initiation of a crack for D16AT alloy. Independently of the acting stresses, the crack is initiated for the constant limit degree of saturation of the DP $D^{*} \cong 0.366$ (as $\bar{N} \rightarrow 1$ ), which confirms the efficiency of application of this characteristic for the prediction of the service life of D16AT alloy in fatigue. 


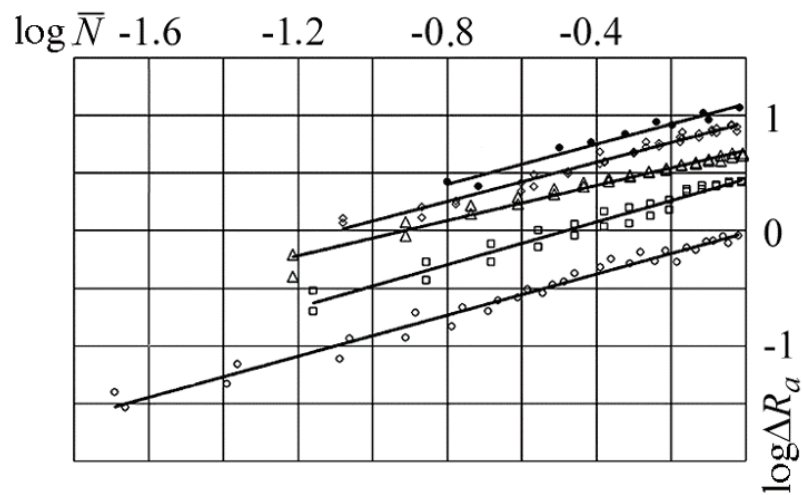

Fig. 5. Dependences of the increment of the surface roughness on the number of loading cycles prior to the initiation of a crack for various stresses $\sigma_{\max }$ (see the notation in Fig. 4).

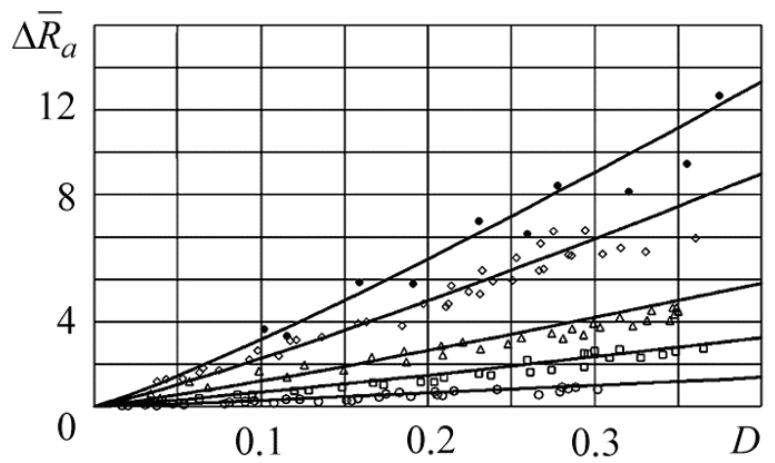

Fig. 6. Experimental data (symbols) and the dependences of the increment of the surface roughness on the saturation of the DP plotted according to relation (8) (lines) for various values of $\sigma_{\max }$ (see Fig. 4).

Roughness. Under cyclic loading, the DP develops not only in the plane but also in the direction normal to the surface of the specimen. The level of roughness increases with the number of loading cycles by an order of magnitude. The dependences of the relative increment of roughness $\Delta \bar{R}_{a}=\Delta R_{a} / R_{a 0}$ on the relative number of loading cycles $\bar{N}$ for various levels of stresses in a cycle presented on the double logarithmic coordinates have the form of straight lines with almost equal slopes (Fig. 5) and can be described by the following function:

$$
\Delta \bar{R}_{a}=7 \cdot 10^{-4} \sigma_{\max }\left(\sigma_{\max }-34\right) \bar{N}^{0.9}
$$

By using the empirical relations (6) and (7), we get the following dependence for the description of the development of the DP on the surface of the cladding layer under cyclic loading in three dimensions [namely, in the plane (described by the parameter $D$ ) and along the normal to the surface (described by the parameter $\left.\left.\Delta \bar{R}_{a}\right)\right]$ :

$$
\Delta \bar{R}_{a}=2.17 \cdot 10^{-3} \sigma_{\max }\left(\sigma_{\max }-34\right) D^{1.125}
$$

where $60 \mathrm{MPa} \leq \sigma_{\max } \leq 150 \mathrm{MPa}$. The experimental data on the development of the DP for various levels of stresses in loading cycles reveal the adequacy of Eq. (8) (see Fig. 6). 


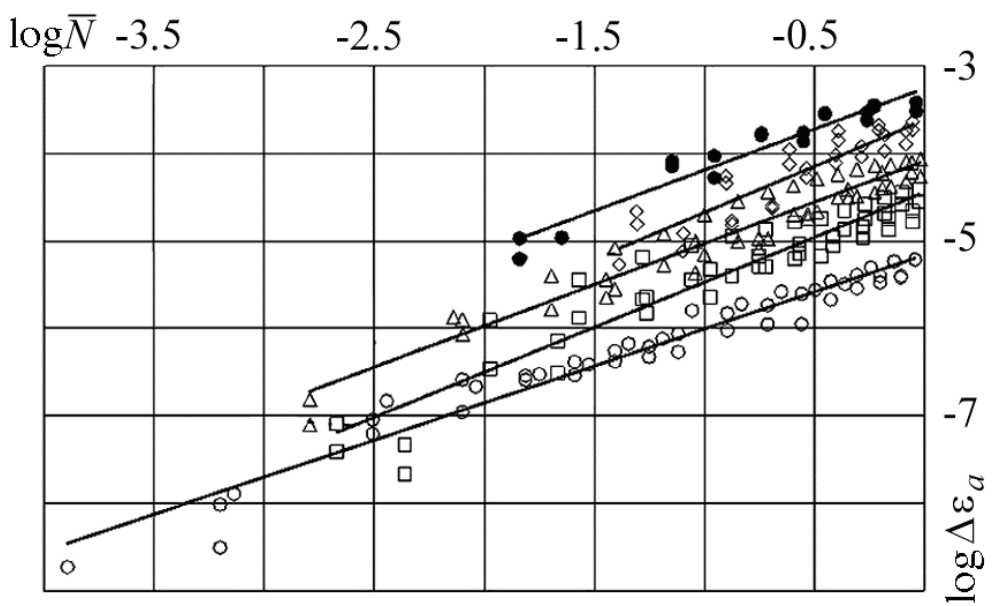

Fig. 7. Dependences of the level of plastic strains on the surface on the number of loading cycles for various levels of stresses $\sigma_{\text {max }}$ (see Fig. 4).

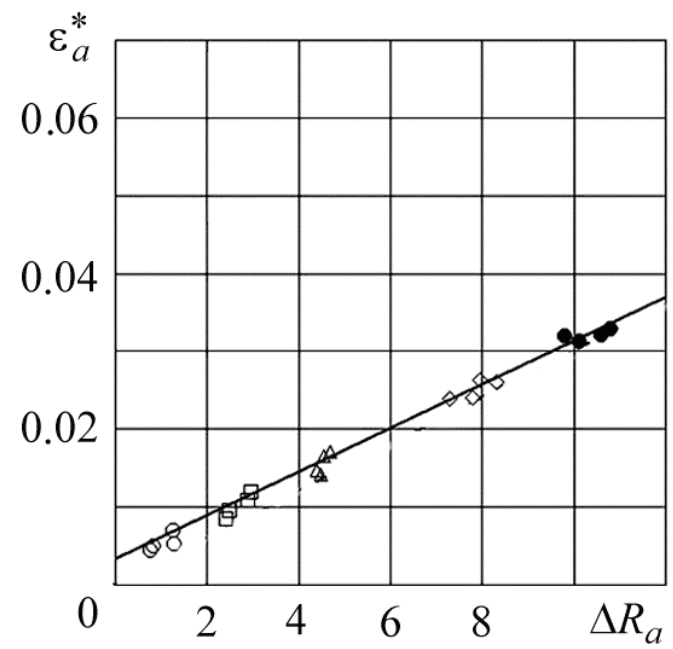

Fig. 8. Comparison of the experimental data (symbols) with the theoretical results [the solid line is plotted according to relation (11)] on the limit values of plastic strains and the relative surface roughness (prior to the formation of a fatigue crack $0.5 \mathrm{~mm}$ in length) (see Fig. 4).

\section{Plastic Deformation of the Surface}

Under cyclic loading, the surface area of the cladding layer increases and its irreversible deformation becomes more pronounced. The dependences of the level of strains $\varepsilon_{a}$ on the number of loading cycles are approximated by a power function and strongly depend on the acting stresses (Fig. 7). Under the stresses $\sigma_{\max }=$ 60-150 MPa, the plastic strains change by three orders of magnitude. The obtained results can be generalized by the dependence

$$
\varepsilon_{a}=1.67 \cdot 10^{-6} \sigma_{\max }^{2} \bar{N}^{0.96}
$$


Excluding the parameter $\bar{N}$ from expressions (9) and (6) and finding $\sigma_{\max }$ from relation (8), we arrive at the generalized dependence of the plastic strains formed on the surface on the parameters $D$ and $\Delta \bar{R}_{a}$ :

$$
\varepsilon_{a}=1.614 \cdot 10^{-3}\left(1+\sqrt{1+1.6 \cdot \Delta \bar{R}_{a} D^{-1.125}}\right)^{2} D^{1.2}
$$

Further, substituting the limit value of saturation of the DP $D^{*} \cong 0.366$ obtained from relation (6) in relation (10) for $\bar{N}=1$, we get the following relationship between the critical values of the parameters $\varepsilon_{a}^{*}$ and $\Delta \bar{R}_{a}^{*}$ for the time of formation of the fatigue crack:

$$
\varepsilon_{a}^{*}=4.825 \cdot 10^{-4}\left(1+\sqrt{1+4.963 \cdot \Delta \bar{R}_{a}^{*}}\right)^{2}
$$

Note that dependence (11) is plotted on the basis of the generalization and approximation of the experimental data on the variations of the parameters $\varepsilon_{a}, D$, and $\Delta \bar{R}_{a}$ as functions of the number of loading cycles and these data are characterized by a natural spread of values. Therefore, the condition $\varepsilon_{a}^{*}=0$ for $\Delta \bar{R}_{a}^{*}=0$ is not determining for the analyzed dependence. Dependence (11) is in good agreement with the experimental values of the parameters $\varepsilon_{a}^{*}$ and $\Delta \bar{R}_{a}^{*}$ corresponding to the formation of the fatigue crack (Fig. 8), which means that this dependence is adequate.

\section{CONCLUSIONS}

The DP is formed and develops on the surface of the cladding layer of D16AT alloy under cyclic loading. The surface area with traces of the DP increases with the number of loading cycles, which is accompanied by the increase in its roughness and plastic strains. The initiation of a fatigue crack corresponds to the limit value of saturation of the DP $D^{*} \cong 0.366$, which does not depend on the level of applied stresses. The time of formation of the fatigue crack is also determined by the limit values of plastic strains $\varepsilon_{a}^{*}$ and the parameter of roughness of the surface $\Delta \bar{R}_{a}^{*}$. The relationship between these limit values of the DP is independent of the maximum stress in a loading cycle.

\section{REFERENCES}

1. V.E. Panin and A. V. Panin, "Effect of the surface layer in a deformed solid body," Fiz. Mezomekh., 8, No. 5, 7-15 (2005).

2. E. E. Zasimchuk, A. I. Radchenko, and M. V. Karuskevich, "Single crystals as an indicator of fatigue damage," Fatigue Fract. Eng. Mater. Struct., 15, No. 12, 1281-1283 (1992).

3. S. R. Ignatovich, M. V. Karuskevich, and O. M. Karuskevich, UR, Patent No. 29683 (2008).

4. S. Ignatovich, M. Karuskevich, and T. Maslak, "Computer-aided optical method for aircraft's components fatigue life estimation," in: Abstr. and Proc. of the 17th Europ. Conf. on Fracture (September 2-5, 2008, Brno), Brno (2008) (on the CD ROM), pp. 23082313.

5. M. V. Karuskevich, E. Yu. Korchuk, A. S. Yakushenko, and T. Maslak, "Estimation of the accumulated fatigue damage by saturation and fractal dimension of the deformation relief," Strength Mater., 40, No. 6, 693-698 (2008). 
6. S. R. Ignatovich, M. V. Karuskevich, T. P. Maslak, and S. S. Yutskevich "Evolution of the deformation pattern of the cladding layer of D16AT aluminum alloy in fatigue," in: Proc. of the Internat. Sci.-Eng. Conf. "Damage of Materials in the Process of Operation, Methods of Its Diagnostics and Prediction” (September 21-24, 2009, Ternopil) [in Ukrainian], Ternopil State Technical University, Ternopil (2009), pp. 47-53.

7. S. R. Ignatovich, M. V. Karuskevich, T. P. Maslak, and S. S. Utskevich, "Deformation surface pattern as an indicator of fatigue damage to aviation alloys based on aluminum," in: Proc. of the 3rd Internat. Conf. "Deformation and Fracture of Materials and Nanomaterials," (October 12-15, 2009, Moscow) [in Russian], Vol. 1, Interkontakt, Nauka, Moscow (2009), pp. 53-54.

8. http://www.nau.edu.ua/en/Science/GotoviRozrobky/MicronAlpha. 